\title{
Criatividade e Inteligência em Crianças: Habilidades Relacionadas?
}

\author{
Tatiana de Cássia Nakano ${ }^{1}$ \\ Pontifícia Universidade Católica Campinas
}

\begin{abstract}
RESUMO - Diante dos questionamentos que persistem acerca da relação entre criatividade e inteligência, a pesquisa teve por objetivo investigar esses construtos junto a crianças, 90 estudantes do Ensino Fundamental (44 do sexo feminino e 46 do sexo masculino), com idades entre sete e 12 anos, que responderam a um teste não verbal de desenvolvimento cognitivo (Desenho da Figura Humana) e a um teste de criatividade (Teste de Criatividade Figural Infantil). Os resultados demonstraram que o desempenho no teste cognitivo relacionou-se significativamente com o desempenho no teste de criatividade $(\mathrm{r}=0,47 ; \mathrm{p}<0,01)$, de forma que, no presente estudo, criatividade e inteligência encontram-se relacionadas. Conforme esperado, ambos os construtos mostraram-se sensíveis à influência da idade, sendo que a variável gênero não se mostrou significativa.
\end{abstract}

Palavras-chave: criatividade, inteligência, avaliação, ensino fundamental.

\section{Creativity and Intelligence in Children: Related Abilities?}

\begin{abstract}
Considering the questions that still remain concerning the relationship between creativity and intelligence, this study aimed to investigate these constructs in children. A sample of 90 elementary school students (44 girls and 46 boys) with ages between 7 and 12 years responded to a nonverbal test of cognitive development (Human Figure Drawing Test) and a creativity test (Test of Figural Creativity for Children). Results on the cognitive test showed a significant correlation with the outcome of the creativity test $(r=0.47, p<0.01)$, indicating that, in the present study, creativity and intelligence are related. As expected, creativity and cognitive development were shown to be characteristics that increase according to age. On neither of the tests significant differences between boys and girls were observed.
\end{abstract}

Keywords: creativity, intelligence, assessment, elementary school.

Nos últimos anos vive-se um novo ciclo de interesse pelo estudo da inteligência e suas medidas, especialmente porque elas são frequentemente relacionadas a comportamentos socialmente valorizados, tais como desempenho acadêmico, desenvolvimento profissional, traços de personalidade, entre outros (Santos, Noronha \& Sisto, 2005). A criatividade, por outro lado, também tem sido uma característica cada vez mais valorizada na literatura devido à sua capacidade de promover o desenvolvimento completo e sadio do indivíduo (Wechsler, 2008). Diante da importância reconhecida desses dois construtos, a literatura cientifica há bastante tempo vem buscando investigar a existência de relação entre criatividade e inteligência, cujos estudos tiveram início há quase 50 anos a partir das pesquisas conduzidas por Wallach e Kogan (1965), posteriormente também investigadas por Torrance (1980), a partir das quais começa a ficar claro que o conhecimento total do potencial individual somente pode ser descoberto quando esses dois construtos são estudados conjuntamente. Por outro lado, também nessa época, os estudos de Getzels e Jackson (1962), bastante importantes, assumem os dois construtos como totalmente diferentes e independentes, de forma que, apesar de ser um tema bastante estudado, as investigações atuais seguem questionando a existência dessa relação e o nível da associação, além da sua estabilidade no tempo e

1 Endereço para correspondência: Puc, Campus Universitário, Departamento de Pós-Graduação em Psicologia, Avenida John Boyd Dunlop, Jardim Ipaussurama. Campinas, SP. CEP 13060-904. E-mail: tatiananakano@hotmail.com possibilidade de generalização para populações distintas (Elisondo \& Donolo, 2010).

Embora, desde então, esses questionamentos venham sendo bastante pesquisados, ainda não se pode encontrar, na literatura, um consenso sobre a questão, de forma ser comum verificar a existência de algumas vertentes e modelos explicativos muitas vezes até contraditórios. Enquanto alguns autores afirmam a existência de uma alta relação entre criatividade e inteligência, outros defendem a independência entre esses dois construtos (Hattie \& Rogers, 1986). Outra explicação apóia-se no fato de que essa relação pode não ser simplesmente do tipo linear, mas que estaria relacionada a partir de um certo nível de inteligência (threshold theory), na qual um nível moderado de inteligência seria necessário para que a criatividade ocorra.. Assim, o que se pode notar é que a ligação entre criatividade e inteligência tem sido extensivamente investigada, levando a diferentes resultados de acordo com o tipo de estudo, sendo que, os achados, aparentemente contraditórios, podem ser explicados, em parte, pela heterogeneidade das medidas empregadas e tipo de população estudada.

Em relação à primeira corrente, que afirma a existência de relação entre criatividade e inteligência, um modelo explicativo bastante difundido segue a definição de um fator $\mathrm{G}$ de inteligência baseado nas idéias de Binet, o qual envolveria basicamente a capacidade de conceituar e explorar abstrações com facilidade, sendo a criatividade conceituada como a capacidade de reconhecer idéias novas e originais e a exploração das mesmas (Nogueira \& Pereira, 2008), de 
forma que nesta linha de pensamento a realização criadora requer então criatividade e inteligência conjuntamente. Os autores que partilham esta opinião apresentam como principal argumento as investigações realizadas com adultos eminentes nos quais a criatividade elevada se associa a excelentes resultados em testes de QI.

Nesse sentido, Sternberg (2001) argumenta que pessoas criativas não somente geram um grande número de idéias, mas também analisam-nas, de maneira a discriminar (inteligentemente) entre a melhor e a pior. Assim, a inteligência seria um pré-requisito da criatividade. Neste mesmo sentido Kneller (1971) se posiciona de forma a afirmar que a correlação entre inteligência e criatividade é alta, sem, todavia, ser absoluta. Para o autor, embora não constitua condição suficiente, o QI elevado parece ser, de um modo geral, necessário para a alta criatividade visto que são poucas as pessoas altamente criativas que também não são altamente inteligentes. Dessa maneira esse modelo se ampara na argumentação de que uma pessoa inteligente, mas não criativa, poderia ser capaz de demonstrar grande habilidade para pensamento abstrato, porém produziria poucas idéias originais. Por outro lado uma pessoa criativa, mas sem alta inteligência, seria incapaz de desenvolver plenamente a sua elaboração mental devido à dificuldade em manter, por período prolongado, o pensamento abstrato, de forma que, pelos motivos apresentados, seria ideal a presença de ambos os construtos. A crítica a este modelo se embasa no argumento de que a criatividade ficaria restrita à capacidade de reconhecer a novidade de idéias que a inteligência já produziu.

Os autores que partilham da segunda corrente, segundo a qual a criatividade e inteligência seriam construtos independentes, defendem a idéia de que uma pessoa com alta inteligência pode, ou não, ser altamente criativa, de forma a se afirmar que os dois construtos podem ser separados conceitualmente (Kim, Cramond \& Bandalos, 2006; Sternberg, 1984). A explicação das diferenças poderia situar-se no fato de que "a inteligência tem sido concebida como crucial na adaptação a ambientes existentes, enquanto que a criatividade, a qual envolve a produção de idéias ou produtos novos e úteis, tem sido vista como crucial na modificação e modelagem do ambiente" (Sternberg \& O'Hara, 2000, p. 611). Nesta visão, o QI por si só não é o único fator para a determinação do talento criativo uma vez que a criatividade pode ser vista como construção suficientemente distinta da inteligência, podendo, inclusive, servir como preditor concorrente para a realização escolar (Rindermann \& Neubauer, 2004). Outro argumento bastante utilizado refere-se ao fato de que alguns estudos, tais como os relatados por Getzels e Jackson ou Torrance apontam que os testes de inteligência e criatividade revelam uma baixa correlação entre si (Edwards \& Tyler, 1965).

Como forma de embasar este argumento, pesquisadores apontam resultados de investigações que revelaram a existência de criatividade elevada em sujeitos cujo QI era inferior a 70 e investigações realizadas com crianças de elevado QI associado a um excelente rendimento escolar, mas cujos desempenhos são medianamente criativos (Nogueira \& Pereira, 2008). A mesma opinião é compartilhada por Boden (1999), segundo a qual a inteligência não é condição suficiente para uma criatividade elevada. Alguns estudos que demonstram a não existência de relação podem ser citados, tais como a pesquisa de Wechsler e Richmond (1984) realizada com crianças na qual foram demonstradas fracas relações, não significativas, entre inteligência e criatividade. Resultados similares são encontrados por Edwards e Tyler (1965), cujo estudo com estudantes do Ensino Fundamental encontrou correlação de 0,08 entre uma medida de inteligência e outra de criatividade, ou ainda pesquisa de Russo (2004) que, ao testar dois grupos, um com alto QI e outro com baixo QI, também não encontrou diferenças significativas entre os grupos nos processos de identificação e solução de problemas, avaliados através das variáveis fluência, flexibilidade, elaboração e originalidade.

A terceira vertente trabalha com a suposição de que criatividade e inteligência estariam relacionadas a partir de um certo nível de inteligência (teoria do threshold), segundo a qual seria necessário um nível mínimo de inteligência para que a criatividade possa ocorrer. Esse nível mínimo vem sendo estimado, por vários autores, como um valor de QI em torno de 120, como pode ser verificado na opinião de Kneller (1971, p.21) ao afirmar que "para a alta criatividade se precisa de QI de pelo menos 120”. Opinião similar é compartilhada por Eysenck (1999), segundo o qual a correlação entre as medidas de criatividade e o QI seria certamente não linear, sendo apreciável em níveis de QI acima de 120 ou próximos ou ainda por Preckel, Holling e Wiese (2006) ao afirmarem que a relação entre criatividade e inteligência expressaria correlações altas em QI acima de 120 e baixas correlações em QI abaixo de 120. Para Lubart (2007), as pessoas criativas tendem a ter um QI superior ao da média, frequentemente acima de 120, de forma que valores iguais ou superiores a este facilitariam a entrada em áreas nas quais um trabalho altamente criativo é possível, embora, na opinião do autor, a partir de um certo nível de QI não haveria mais benefício suplementar.

$\mathrm{Na}$ visão dos teóricos dessa corrente, para ter um nível elevado de criatividade seria necessário apresentar pelo menos um certo nível de inteligência e outros componentes como certos traços de personalidade (Lubart, 2007), de modo que se pode afirmar que a pessoa criativa também deveria apresentar uma série de características próprias, tais como questionamento, curiosidade e inconformismo, que refletem a criatividade. Por este motivo, segundo Shaffer (2005), talvez seja possível compreender porquê tantas pessoas com QI alto ou superdotadas não são particularmente criativas ou porquê de tão poucas serem proeminentes.

Dessa forma, o que se pode verificar é que, embora ainda não haja consenso sobre a natureza da relação entre inteligência e criatividade, vários modelos incluem esta última característica como uma dimensão da inteligência ou relacionada a este construto. Neste sentido Sternberg e O`Hara (2000) sintetizam os resultados relatados na literatura científica mostrando que os estudos que buscaram relacionar esses dois construtos hipotetizaram acerca de cinco possíveis respostas: (a) inteligência como super conjunto da criatividade, (b) inteligência como um subconjunto da criatividade, (c) inteligência e criatividade como construtos relacionados, (d) inteligência e criatividade como sendo essencialmente a mesma coisa, isto é, coincidentes, (e) inteligência e criatividade não tendo nenhuma relação entre si. Essa constatação 
nos leva à importância de se refletir sobre o tema e procurar respostas que determinem a existência ou não desta relação, cujas respostas podem enriquecer, por exemplo, as discussões na temática da superdotação. Isto porque, dentro dos modelos mais atuais, esta seria composta por diversas facetas, destacando-se dentre elas, inteligência e criatividade (Feldman, 2000; Gagné, 2005; Jarosewich, Pfeiffer \& Morris, 2002; Kaufman \& Sternberg, 2008; Renzulli, 2008).

Assim, diante do que foi apontado acerca dos questionamentos que ainda persistem na literatura acerca da relação existente os domínios da criatividade e inteligência, a presente pesquisa teve por objetivo investigar esses dois construtos em uma amostra de crianças, verificando se o desempenho em testes específicos de avaliação da criatividade e inteligência estaria relacionado.

\section{Método}

\section{Participantes}

Participaram da pesquisa 90 estudantes de $2^{\circ}$ ao $6^{\circ}$ ano do Ensino Fundamental de uma escola particular situada na capital de um estado pertencente à região nordeste do país, sendo 44 do sexo feminino e 46 do sexo masculino, com idades entre sete e 12 anos (média de 9,33 anos e $\mathrm{DP}=1,49$ ), os quais foram selecionados dentro de uma amostra de conveniência.

\section{Instrumentos}

Teste de Criatividade Figural Infantil (Nakano, Wechsler \& Primi, 2011)

Instrumento no qual os participantes são convidados a comporem desenhos em três atividades, a partir do fornecimento de estímulos pouco definidos. As características avaliadas pelo instrumento são 12, corrigidas em cada uma das três atividades: Fluência, Flexibilidade, Elaboração, Originalidade, Expressão de Emoção, Fantasia, Movimento, Perspectiva Incomum, Perspectiva Interna, Uso de Contexto, Extensão de Limites e Títulos Expressivos. Sua correção permite a obtenção de pontuações em quatro fatores: Fator 1 -Enriquecimento de Idéias (capacidade ver a situação de uma forma mais detalhada, por um ponto de vista diferente; Fator 2 - Emotividade (composto por características que envolvem o uso de recursos criativos ligados à uma percepção mais emocional); Fator 3 - Preparação Criativa (separa basicamente algumas características avaliadas na primeira atividade do teste, funcionando como um aquecimento para a realização das demais atividades); Fator 4 - Aspectos Cognitivos (composto por características criativas que fazem uso de recursos cognitivos, que envolvem a busca de soluções diferenciadas, originais e que vão além dos limites estabelecidos). Também gera uma pontuação total no instrumento, resultado da soma dos quatro fatores. Importante salientar que a pontuação é baseada em aspectos da criatividade e não na qualidade artística.
O instrumento foi embasado no teste figurativo de Torrance (Torrance Test of Figural Thinking), embora importantes e substanciais ajustes tenham sido feitas: (1) os estímulos que compõem o instrumento foram alterados, tendo sido selecionados a partir de desenhos feitos por crianças brasileiras; (2) as instruções foram modificadas de forma a evidenciar as tarefas e atender à necessidade de adaptação cultural; (3) a ocorrência de cada característica criativa passou a ser considerada separadamente em cada uma das três atividades, diferentemente do teste de Torrance no qual as características criativas, ainda que pontuadas separadamente em cada atividade, são somadas e consideradas em sua totalidade, independente da atividade em que foi pontuada; (4) a característica de combinação foi retirada devido à baixa ocorrência na amostra brasileira; (5) o sistema de interpretação é baseado em um modelo de quatro fatores (enriquecimento de idéias, aspectos emocionais, preparação criativa e aspectos cognitivos) extraído após realização de análise fatorial com uma amostra composta por 1500 crianças brasileiras, diferentemente do modelo original, composto por dois índices (aspectos cognitivos e aspectos cognitivos mais emocionais).

Pesquisas investigando as evidências de validade e precisão do Teste de Criatividade Figural Infantil foram realizadas (Nakano, 2003) e indicaram valores entre $0,81 \mathrm{e}$ 0,94 de correlação para validade concorrente com o Teste Figural de Torrance (com nível de significância de $\mathrm{p} \leq 0,001$ para todas as características avaliadas) e índices de precisão por meio do teste e reteste entre 0,84 e 0,95 ( $p \leq 0,001)$, confirmando evidências psicométricas favoráveis para uso em amostras brasileiras (Nakano \& Wechsler, 2006a). Outros estudos ainda mostraram que os resultados sofrem influência significativa das variáveis série (Nakano \& Wechsler, 2006b), região e tipo de escola (Nakano, Wechsler \& Primi, 2011).

\section{Teste Desenho da Figura Humana (Wechsler, 2003)}

No teste é solicitada à criança a elaboração de dois desenhos (desenho de uma figura feminina e de uma figura masculina). Constitui-se como uma medida não verbal do desenvolvimento cognitivo, uma vez que "inicialmente as crianças desenham o que sabem e não o que vêem, sendo que, com o desenvolvimento, a criança tentará, cada vez mais, representar os objetos como os vê, surgindo gradualmente os conceitos de tamanho, proporção, posição relativa das partes, relação espacial e outros" (Bandeira, Costa \& Arteche, 2008, p.332). O instrumento mostra-se bastante adequado para uso em crianças de pouca idade devido à familiaridade com a tarefa a ser executada, de maneira que a escolha pela utilização de um teste cognitivo não verbal deu-se pela similaridade da tarefa (realização de desenhos) entre os dois instrumentos.

Os desenhos são analisados de acordo com a presença ou ausência de detalhes ou itens, os quais são somados e dão origem ao total de pontos brutos para o desenho do homem e o total de pontos brutos para o desenho da mulher. As pontuações de cada desenho e a pontuação total (soma dos dois) darão origem a percentis e uma classificação de acordo com a média esperada para a sua faixa de desenvolvimento, de forma que a interpretação dos pontos obtidos pela criança permite situá-la em relação ao seu grupo etário comparativo (de acordo com tabelas etárias com intervalos de seis meses). 
O manual traz resultados de estudos psicométricos que apontaram um coeficiente de 0,75 para a figura feminina e um coeficiente de 0,69 para a figura masculina em relação à estabilidade temporal, assim como um coeficiente alpha para o desenho feminino entre 0,77 e 0,85 e entre 0,83 a 0,89 para o desenho masculino, para consistência interna, em ambos os sexos. A precisão dos avaliadores indicou altas concordâncias entre os juízes que variaram entre 0,95 a 0,98 para a figura masculina e de 0,93 a 0,97 para a figura feminina (Wechsler, 2003).

\section{Procedimento}

Após autorização do Comitê de Ética em Pesquisa e assinatura dos termos de consentimentos pelos pais das crianças, os instrumentos foram aplicados de forma coletiva em sala de aula, em uma única aplicação, que se iniciou com o Teste de Criatividade devido ao fato deste instrumento ter tempo controlado para resposta. Após o término os estudantes receberam o Teste Desenho da Figura Humana, sem tempo determinado para o término da atividade.

Os resultados brutos foram utilizados com a finalidade de comparação de desempenho. Fez-se uso da estatística descritiva, através das médias e desvios padrão de cada instrumento, considerando-se a divisão da amostra por sexo e idade, além da análise da variância para verificar a influência dessas variáveis nos desempenhos. Os dados também foram comparados por meio da Correlação de Pearson para verificar a relação entre o desempenho nos dois instrumentos.

\section{Resultados e Discussão}

Inicialmente são fornecidas as médias e desvios padrão por sexo e idade para o Teste de Criatividade Figural Infantil (quatro fatores e pontuação total) e para o Desenho da Figura Humana (desenho da figura feminina, masculina e total).

Em relação ao teste de criatividade os resultados indicaram que o sexo masculino obteve médias mais altas que o feminino na maior parte das medidas (Fator 1 - Enriquecimento de Idéias, Fator 3 - Preparação Criativa, Fator 4 - Aspectos Cognitivos e na pontuação total). A exceção ocorre em relação ao Fator 2 (Emotividade), no qual o sexo feminino desempenhou-se melhor.

Igualmente, a análise do desempenho criativo de acordo com a idade aponta para maiores médias obtidas pelos participantes de 12 anos na maior parte das medidas criativas (Fator 1 - Enriquecimento de Idéias, Fator 3 - Preparação Criativa e pontuação total), fato que pode estar sinalizando um aumento no nível de criatividade de acordo esta variável. Participantes de 11 anos pontuaram melhor no Fator 2 (Emotividade) e os de 8 anos no Fator 4 (Aspectos Cognitivos). Participantes de 7, 9 e 10 anos não obtiveram destaque em nenhuma medida criativa.

Analisando-se o instrumento de avaliação do desenvolvimento cognitivo, o DFH, os dados indicam que o sexo masculino obteve melhor desempenho, apresentando médias mais altas nas três medidas do teste (desenho da figura feminina, masculina e total). Interessantemente, podemos ver que ambos os sexos obtêm melhor pontuação quando desenham a figura masculina. A análise do desempenho considerando a idade mostra que as pontuações nas três medidas aumentam juntamente com essa variável, sendo esse resultado previsto, uma vez que se espera que a maturidade conceitual e a percepção de mundo da criança acompanhem o aumento da sua idade.

Os dados apresentados na Tabela 1 ainda nos levam a algumas reflexões. Corroborando os resultados que eram esperados, criatividade e desenvolvimento cognitivo mostraram ser características que aumentam de acordo com a idade, indicando ganhos na amostra estudada. Estes resultados vão ao encontro tanto dos dados fornecidos no manual do Teste de Criatividade Figural Infantil (Nakano, Wechsler \& Primi, 2011) quanto no manual do Desenho da Figura Humana (Wechsler, 2003). Em relação à criatividade, a constatação também converge com a percepção de Torrance (1976), segundo o qual a curva de desenvolvimento para a capacidade criativa sobe gradualmente nos primeiros anos de escola atingindo um nível bastante alto aos onze anos de idade e ainda com outras pesquisas desenvolvidas com o instrumento de criatividade (Nakano \& Wechsler, 2006a, 2006b). Em relação à inteligência, Wechsler e Schelini (2006) apontaram aumento significativo no desempenho em todos os tipos de habilidades cognitivas investigadas no período dos 7 aos 18

Tabela 1. Médias e Desvios Padrão por Idade e Sexo no Teste de Criatividade Figural Infantil e Desenho da Figura Humana

\begin{tabular}{|c|c|c|c|c|c|c|c|c|c|c|c|c|c|c|c|c|}
\hline \multirow[t]{2}{*}{ Variável } & \multicolumn{2}{|c|}{ F1 } & \multicolumn{2}{|c|}{$\mathrm{F} 2$} & \multicolumn{2}{|c|}{ F3 } & \multicolumn{2}{|c|}{ F4 } & \multicolumn{2}{|c|}{ Criat. total } & \multicolumn{2}{|c|}{ Fig. Fem. } & \multicolumn{2}{|c|}{ Fig. Masc. } & \multicolumn{2}{|c|}{ DFH total } \\
\hline & $\mathrm{M}$ & DP & M & DP & $\mathrm{M}$ & DP & M & DP & $\mathrm{M}$ & DP & $\mathrm{M}$ & DP & $\mathrm{M}$ & DP & $\mathrm{M}$ & DP \\
\hline 7 anos & 25,3 & 15,6 & 2,8 & 5,7 & 4,9 & 3,2 & 27,3 & 12,9 & 60,4 & 28,1 & 20,8 & 6,0 & 21,6 & 3,7 & 42,5 & 9,0 \\
\hline 8 anos & 38,8 & 19,1 & 0,9 & 1,9 & 7,1 & 4,0 & 44,1 & 17,8 & 91,0 & 33,7 & 26,00 & 7,28 & 27,41 & 5,40 & 53,41 & 11,88 \\
\hline 9 anos & 43,0 & 20,9 & 1,7 & 4,0 & 6,9 & 9,3 & 43,5 & 9,3 & 95,2 & 28,9 & 26,71 & 6,67 & 29,57 & 7,36 & 56,19 & 12,73 \\
\hline 10 anos & 57,2 & 26,5 & 6,8 & 8,1 & 7,0 & 3,5 & 42,3 & 10,4 & 113,5 & 30,3 & 28,57 & 5,99 & 29,79 & 7,46 & 58,36 & 13,11 \\
\hline 11 anos & 56,1 & 22,9 & 9,6 & 6,7 & 7,9 & 5,1 & 40,1 & 14,9 & 113,8 & 34,9 & 30,57 & 3,99 & 32,14 & 4,17 & 62,71 & 6,92 \\
\hline 12 anos & 61,6 & 8,2 & 6,0 & 5,7 & 11,0 & 2,8 & 37,0 & 17,0 & 115,6 & 22,2 & 31,20 & 6,94 & 35,00 & 4,69 & 66,20 & 10,68 \\
\hline Feminino & 44,0 & 22,9 & 4,9 & 6,3 & 6,5 & 4,5 & 39,8 & 14,5 & 95,4 & 35,9 & 27,00 & 7,22 & 28,05 & 6,47 & 55,05 & 13,06 \\
\hline Masculino & 48,1 & 23,8 & 4,3 & 6,6 & 7,7 & 4,2 & 40,4 & 14,3 & 100,7 & 34,7 & 27,46 & 6,19 & 30,00 & 6,77 & 57,41 & 11,95 \\
\hline
\end{tabular}


anos de idade, concordando, segundo as autoras, com dados da literatura internacional.

Uma segunda constatação importante, ainda discutindo-se a questão da idade, refere-se ao fato de que os resultados indicaram uma maior pontuação das crianças de oito anos no fator referente ao pensamento divergente. Uma possível explicação apóia-se na percepção que crianças mais novas ainda não se censuram, permitindo que todas as idéias pensadas sejam valorizadas e expressas. Essa habilidade vai se perder, segundo Torrance (1976), mais ou menos por volta do quarto ano escolar, quando ocorre o estágio de realismo, ou idade da turma, durante o qual começa a surgir uma conformidade aos padrões do grupo de iguais, desaparecendo muito do pensamento e da ação livre. Neste período os símbolos tornam-se rígidos e os papéis sexuais adquirem grande significação, de forma que poucas são as crianças capazes de resistir à pressão de conformar-se com normas comportamentais nesse estágio. A maioria tende a ter sua liberdade reduzida, além da liberdade de comunicação, principalmente aquela relacionada a idéias originais. Neste sentido, ainda segundo o autor, notar-se-ia uma mudança de comportamento, que passaria da insaciável curiosidade dos alunos do segundo ano sobre o "por que" e o "como" das coisas para uma procura por explicações por volta do terceiro e quarto ano (Torrance, 1976). Por este motivo pode-se explicar a queda gradual que o fator relacionado ao pensamento divergente vai apresentando ao longo dos anos, conforme disposto na Tabela 1 .

Diante das aparentes diferenças encontradas em relação ao desempenho dos participantes, a influência das variáveis sexo e idade foi investigada a partir da Análise Multivariada da Variância, dado que pode ser visualizado na Tabela 2. Os resultados indicaram que a idade do participante influencia de forma significativa todas as medidas do teste de desenvolvimento cognitivo e a maior parte das medidas do teste de criatividade, com exceção do Fator 3 (Preparação Criativa). Essa única exceção pode ser explicada devido à constituição desse fator (que ao avaliar somente as características criativas na Atividade 1 do instrumento, torna-se diferente das demais atividades uma vez que permite a criação de uma única resposta, fazendo com que, provavelmente não hajam muitas diferenças entre os participantes devido a essa limitação imposta pela natureza da atividade).

Ainda na Tabela 2 pode-se verificar que as variáveis sexo e a interação entre sexo e idade não se mostraram significativas, sendo que a constatação da não diferenciação entre homens e mulheres, vem reforçar uma das posições téoricas defendidas na literatura acerca da hipótese de não existência de diferenças na criatividade devido ao gênero. Autores como Aranha (1997) apoiam-se na ideia de que os indivíduos criativos escapariam, em certa medida, ao rígido estereótipo dos papéis em função do gênero, fato que seria explicado, a partir da questão da androgenia psicológica, que se refere à capacidade que uma pessoa tem para ser ao mesmo tempo agressiva e protetora, sensível e rígida, dominante e submissa, independente do gênero ao qual pertença. Deste modo, pessoas com alto nível de criatividade assemelhariam-se mais entre si do que em função do gênero, opinião também compartilhada por outros autores, tais como Candeias (2008), De La Torre (2005) e Montuori e Purser (1995). Em relação à inteligência, a questão da diferença de gênero também vem sendo amplamente discutida, ainda que nenhum consenso tenha sido atingido (Nisbett et al., 2012). Por um lado, autores afirmam a existência de diferenças em relação a habilidades específicas (Brunner, Krauss \& Kunter, 2008; Dai, Ryan, Paolo \& Harrington, 1991; Goldbeck, Daseking,

Tabela 2. Análise Multivariada da Variância por Sexo e Idade para o Teste de Desenvolvimento Cognitivo e Teste de Criatividade

\begin{tabular}{|c|c|c|c|c|}
\hline Variável & Dependente & $\begin{array}{l}\text { Médias ao } \\
\text { quadrado }\end{array}$ & $\mathrm{F}$ & Signif. \\
\hline \multirow[t]{8}{*}{ sexo } & Fig_feminina & 8,25 & 0,22 & 0,64 \\
\hline & Fig_masculina & 13,90 & 0,39 & 0,54 \\
\hline & DFH_total & 0,605 & 0,01 & 0,95 \\
\hline & F1_criatividade & 10,73 & 0,02 & 0,88 \\
\hline & F2_criatividade & 8,44 & 0,26 & 0,61 \\
\hline & F3_criatividade & 5,71 & 0,29 & 0,59 \\
\hline & F4_criatividade & 21,99 & 0,12 & 0,73 \\
\hline & Criativ. Total & 7,12 & 0,01 & 0,93 \\
\hline \multirow[t]{8}{*}{ idade } & Fig_feminina & 117,59 & 3,06 & $0,01^{*}$ \\
\hline & Fig_masculina & 132,71 & 3,69 & $0,01^{*}$ \\
\hline & DFH_total & 493,10 & 3,93 & $0,003^{*}$ \\
\hline & F1_criatividade & 1977,52 & 4,21 & $0,002 *$ \\
\hline & F2_criatividade & 173,03 & 5,34 & $0,001^{*}$ \\
\hline & F3_criatividade & 19,70 & 1,01 & 0,42 \\
\hline & F4_criatividade & 572,04 & 3,08 & $0,01^{*}$ \\
\hline & Criativ. Total & 4564,40 & 4,45 & $0,001 *$ \\
\hline \multirow[t]{8}{*}{ sexo $\mathrm{x}$ idade } & Fig_feminina & 23,12 & 0,60 & 0,70 \\
\hline & Fig_masculina & 9,02 & 0,25 & 0,94 \\
\hline & DFH_total & 54,05 & 0,43 & 0,83 \\
\hline & F1_criatividade & 134,87 & 0,29 & 0,92 \\
\hline & F2_criatividade & 26,21 & 0,81 & 0,55 \\
\hline & F3_criatividade & 8,05 & 0,41 & 0,84 \\
\hline & F4_criatividade & 206,78 & 1,11 & 0,36 \\
\hline & Criativ. Total & 446,02 & 0,44 & 0,82 \\
\hline
\end{tabular}


Tabela 3. Correlação de Pearson entre o Teste de Criatividade Figural Infantil e o Teste Desenho da Figura Humana

\begin{tabular}{cccccccccc}
\hline Variável & $\begin{array}{c}\text { Correl. } \\
\text { Pearson }\end{array}$ & Fig Fem & Fig Masc & DFH total & F1 criat. & F2 criat. & F3 criat. & F4 criat. & $\begin{array}{c}\text { Criat. } \\
\text { total }\end{array}$ \\
\hline Fig. Fem & Correl. & 1 & $0,76(* *)$ & $0,94(* *)$ & $0,47(* *)$ & 0,16 & $0,27(*)$ & 0,20 & $0,45(* *)$ \\
Fig. Masc. & Correl. & $0,76(* *)$ & 1 & $0,94(* *)$ & $0,45(* *)$ & 0,19 & 0,18 & 0,18 & $0,43(* *)$ \\
DFH total & Correl. & $0,94(* *)$ & $0,94(* *)$ & 1 & $0,49(* *)$ & 0,19 & $0,23(*)$ & 0,20 & $0,47(* *)$ \\
F1 criat. & Correl. & $0,47(* *)$ & $0,45(* *)$ & $0,49(* *)$ & 1 & $0,25(*)$ & $0,48(* *)$ & $0,40(* *)$ & $0,93(* *)$ \\
F2 criat. & Correl. & 0,16 & 0,19 & 0,19 & $0,25(*)$ & 1 & 0,13 & $-0,10$ & $0,33(* *)$ \\
F3 criat. & Correl. & $0,27(*)$ & 0,18 & $0,23(*)$ & $0,48(* *)$ & 0,13 & 1 & $-0,05$ & $0,45(* *)$ \\
F4 criat. & Correl. & 0,20 & 0,18 & 0,20 & $0,40(* *)$ & $-0,09$ & $-0,05$ & 1 & $0,65(* *)$ \\
Criat. total & Correl. & $0,45(* *)$ & $0,43(* *)$ & $0,47(* *)$ & $0,93(* *)$ & $0,33(* *)$ & $0,45(* *)$ & $0,65(* *)$ & 1 \\
\hline
\end{tabular}

$* * \mathrm{p} \leq 0,01 ;{ }^{*} \mathrm{p} \leq 0,05$

Hellwig-Brida, Waldmann \& Petermann, 2010; Irwing, 2012; Ramstedt \& Rammsayer, 2002), por outro, resultados de estudos têm demonstrado que homens e mulheres não apresentam níveis diferentes de habilidade geral (Camarata \& Woodcock, 2006; Savage-McGlynn, 2012).

Uma terceira análise teve por finalidade verificar a relação existente entre a medida de criatividade e a medida de inteligência, tendo se constituído no objetivo maior do estudo, para o qual empregou-se a Correlação de Pearson, cujos resultados encontram-se na Tabela 3.

De acordo com a Tabela podemos verificar que o resultado total no teste de desenvolvimento cognitivo relaciona-se significativamente com o resultado total no teste de criatividade $(\mathrm{r}=0,47 ; \mathrm{p} \leq 0,001)$, de forma que se pode afirmar que, no presente estudo, criatividade e inteligência encontram-se moderadamente relacionadas. Deve-se atentar ao fato de que tal valor pode ter sido influenciado pela similaridade da tarefa a ser realizada nos dois instrumentos (desenhos), visto que outros estudos comparando o teste de criatividade com outras medidas de inteligência apresentaram valores mais baixos de correlação: 0,22 com a versão infantil da Bateria de Provas de Raciocínio (Nakano \& Brito, no prelo) ou ainda ausência de correlação significativa, tanto com a versão padrão (Chiodi, Nakano \& Wechsler, 2011a) quanto ampliada da Bateria Woodcock-Johnsson III (Chiodi, Nakano $\&$ Wechsler, 2011b). Ainda de acordo com a Tabela 3 pode-se verificar que a pontuação total no DFH se relaciona de forma significativa com o Fator 1 (Enriquecimento de Idéias) e Fator 3 (Preparação Criativa), ao passo que a pontuação total no Teste de Criatividade Figural Infantil relaciona-se significativamente com a pontuação na figura feminina e com a figura masculina.

A constatação de existência de correlação concorda com estudos encontrados na literatura, os quais têm demonstrado que inteligência e criatividade podem ser considerados construtos diferentes, porém relacionados entre si. Uma revisão mostra que resultados moderados de correlação também são apontados em diversas pesquisas. Runko e Mraz (1992), por exemplo, relataram correlações de 0,58 entre criatividade e inteligência em uma amostra composta por 30 estudantes universitários, Lundsteen (1966) apontou uma relação na ordem de 0,40 encontrada por Wallach e Kogan em um estudo envolvendo estudantes de três grupos: um com alta criatividade e alta inteligência, outro grupo com alta medida em somente um dos construtos e outro com baixas medidas em ambos. Também tendo como foco a investigação das diferenças entre grupos, Preckel, Holling e Wiese (2006) em uma amostra de 1328 estudantes de $4^{\mathrm{a}}$ a $7^{\mathrm{a}}$ séries, classificados em três grupos: baixa, media e alta inteligência (com este ultimo grupo fazendo parte de uma escola especializada em superdotados) fizeram uso do Berlin Structure-of-Intelligence-test (BIS-HB), cujos resultados apontaram que, para a amostra total, inteligência correlacionou-se com criatividade $(\mathrm{r}=0,54)$, com criatividade verbal $(\mathrm{r}=0,51)$, criatividade figural $(\mathrm{r}=0,36)$ e criatividade numérica $(\mathrm{r}=0,38)$.

Nessa mesma linha, Sternberg (2006) também relata ter encontrado uma série de correlações entre medidas de desempenho criativo e testes convencionais de inteligência, sem discriminar exatamente quais seriam os valores, afirmando somente que índices foram maiores quando se relacionou criatividade com inteligência fluída mais do que inteligência cristalizada. A partir da aplicação de diversos instrumentos em universitários, para avaliação da criatividade (Divergent Thinking Fluency, Rated Creativity, Biographical Inventory of Creative Behaviours, Self-rating of creativity) e para avaliação da inteligência (Raven's Advanced Progressive Matrices, General Knowledge Questionnaire), Batey, Furnham e Safiullina (2010) encontraram correlações de 0,29 entre a criatividade total e o resultado no Raven, de 0,27 entre as medidas de pensamento divergente e de 0,23 entre pensamento divergente e conhecimento geral.

Correlação mediana também foi encontrada em um estudo brasileiro, conduzido por Barros, Primi, Miguel, Almeida e Oliveira (2010), no qual uma medida de criatividade (Teste de Criação de Metáforas) apresentou correlação significativa com duas medidas de raciocínio, sendo de 0,31 com raciocínio abstrato e de 0,48 com raciocínio verbal, ao compararem os resultados de dois grupos (um formado por estudantes de um programa de educação de adultos e outro formado por estudantes universitários). Estudo um pouco 
diferente foi conduzido por Silvia (2008a) junto a 242 estudantes universitários que completaram uma série de testes de criatividade (usos incomuns), inteligência (raciocínio fluído, fluência verbal e geração de estratégias), assim como personalidade (fator abertura à experiência por meio de três escalas de auto-relato). $\mathrm{O}$ autor buscou identificar o efeito da inteligência na criatividade, tendo encontrado um fator de primeira ordem cujo efeito médio-grande na criatividade $(0,43)$, sugeriu, segundo o autor, que um fator de inteligência geral mostrou-se mais relevante para a criatividade do que fatores específicos. Por conta desse resultado afirmou que há boas razões para acreditar que a maior parte dos trabalhos mais antigos subestimaram a relação entre criatividade e inteligência, visto que modelos mais atuais, tal como o das equações estruturais, não estava disponível quando grande parte dessas pesquisas foram realizadas, de forma que os dados dos estudos deveriam, em sua opinião, serem reanalisados a fim de que a verdadeira relação entre os construtos possa ser estimada.

Por outro lado, a literatura também apresenta estudos que, embora concordem com a existência de relação entre as medidas, apresentam valores mais baixos de correlação, tal como o desenvolvido por Rindermann e Neubauer (2004) que, fazendo uso de dois testes de inteligência (Raven e Kognitiver Fahigkeits-Test) e dois testes de criatividade (Verbaler Kreativitats-Test e Verwendungs-Test) em 271 estudantes do Ensino Médio relataram correlações de 0,14 entre as medidas de criatividade e inteligência. Outros estudos, tais como o de Elisondo e Donolo (2010) ao investigar grupos diferentes (alunos de nível secundário e universitários em um total de 962 participantes), utilizando-se a prova CREA e o Test Elemental de Inteligencia para avaliar alunos do ensino médio e Matrizes Progressivas de Raven para os universitários, encontraram valores de correlação de 0,16 para os estudantes do ensino médio e de 0,14 para os estudantes do ensino superior.

Outro estudo brasileiro foi desenvolvido por Wechsler, Nunes, Schelini, Ferreira e Pereira (2010) junto a 172 estudantes com idades entre 7 e 18 anos a partir do uso da Bateria Woodcock-Johnson III e os Testes de Pensamento Criativo de Torrance. Os resultados apontaram a existência de correlações significativas, na amostra total, entre inteligência e o índice criativo figural I, que compreende os componentes cognitivos da criatividade figural $(\mathrm{r}=0,22 ; \mathrm{p} \leq 0,01)$ e para o índice criativo figural II, que compreende os componentes cognitivos e emocionais $(\mathrm{r}=0,20 ; \mathrm{p} \leq 0,01)$, fato não ocorreu em relação à criatividade verbal. Entretanto, ao se considerar as correlações entre os grupos com desempenho acima ou abaixo da mediana na Bateria WJ-III, as correlações entre inteligência e criatividade mostraram-se muito baixas, não alcançando significância estatística, de forma a indicar, segundo as autoras, a não existência de um limiar intelectual no qual a criatividade poderia ser esperada. Assim, concluem de forma a afirmar a independência dos construtos, de forma que a inteligência poderia ser considerado um construto separado da criatividade verbal e figurativa.

Estudo que fez uso de métodos mais sofisticados de análises, tais como o das equações estruturais, foi conduzido por Silva (2008b), visando uma reanálise dos dados de Wallach e Kogan. O autor buscou investigar se a relação entre criatividade e inteligência continuaria apresentando-se como não significativa quando esses métodos fossem empregados. Assim, 151 crianças responderam a cinco subtestes de criatividade (usos alternativos, teste de casos, teste de similaridade, teste padrão de significados, teste de significado das linhas), avaliados em relação a originalidade e fluência e dez subtestes de inteligência (três retirados do WISC: vocabulário, arranjo de figuras e blocos, duas medidas de aptidão verbal e quantitativa da School and College Ability Test, além de cinco medidas de rendimento escolar). Os resultados mostraram que, quando consideradas as pontuações totais, criatividade e inteligência apresentaram uma relação modesta, mas significativa de $0,20(\mathrm{p} £ 0,045)$, sendo que a Fluência apresentou uma relação mais forte $(\mathrm{r}=0,21)$ que Originalidade $(\mathrm{r}=0,16)$, entretanto ambas maiores que a correlação relatada por Wallach e Kogan (1965), de 0,09. A análise fatorial confirmatória indicou ainda que um fator de criatividade foi capaz de predizer, significativamente, um fator de inteligência latente, composto pelos 10 escores de inteligência e realização, com uma magnitude de 0,20 . Neste mesmo sentido, outro estudo de Nusbaum e Silvia (2011) investigou, junto a 226 estudantes universitários, o efeito da inteligência fluída na criatividade, por meio das equações estruturais. O modelo encontrado mostrou boa adequação, indicando que a inteligência fluída consegui prever, de forma significativa $\left(\beta=0,45 ; p<0,001 ; R^{2}=0,20\right)$ a criatividade, mais do que os estudos anteriores haviam apontado.

Embora em menor número, uma terceira vertente de pesquisas afirmam a independência entre os construtos. Por exemplo, Aguirre e Conners (2010), usando dois diferentes testes de criatividade (Thinking Creatively in Action and Movement e Multidimensional Stimulus Fluency Measure) e de uma medida de inteligência (Stanford Binet V Abbreviated) em crianças pré-escolares relataram não terem encontrado nenhum resultado significativo entre os escores dos testes. Entretanto os autores argumentam que os resultados do estudo não podem ser generalizados devido ao número limitado de participantes, o qual pode ter influenciado o poder de detectar a correlação, embora acreditem que, se a relação existe, provavelmente ela não seja grande.

Outros tipos de estudos são relatados, tal como pesquisa verificando a influência do nível sócio-econômico na inteligência e criatividade, a qual apontou um desempenho superior, tanto em criatividade quanto em inteligência, obtido pelas crianças de nível socioeconômico mais alto (Aranha, 1997). Uma correlação positiva entre criatividade e inteligência foi encontrada no grupo de nível socioeconômico baixo $(\mathrm{p} \leq 0,01)$ demonstrando, segundo a autora, que uma alta criatividade ou uma alta inteligência pode estar relacionada ao nível social. Outras pesquisas conduzidas com grupos de diferentes níveis intelectuais e criativos também foram realizadas. Maia-Pinto e Fleith (2004) avaliando 77 alunos de um programa de atendimento a alunos superdotados e talentosos encontraram diferenças significativas no nível de criatividade de três grupos, um de alunos com habilidades acadêmicas, outro composto por alunos talentosos e um terceiro por indivíduos habilidosos em ambas as áreas $(\mathrm{p} \leq 0,006)$. As diferenças indicaram que os alunos talentosos apresentaram um desempenho superior aos alunos com habilidades acadêmicas. Outra pesquisa, de Russo (2004), 
também testou dois grupos de estudantes, um composto por alunos superdotados intelectualmente e outro grupo de alunos médios, após treinamento em técnicas de resolução de problemas. Foram encontradas diferenças significativas em relação à criatividade verbal, sendo que, em relação à criatividade figural, estudantes do grupo médio apresentaram aumento no nível de criatividade no pós-teste, ao passo que estudantes com QI elevado apresentaram diminuição, tendo sido percebido que os alunos com alto QI obtiveram uma baixa pontuação e demoraram mais tempo para terminar a atividade.

Por fim, uma terceira análise a ser relatada no presente estudo consistiu na utilização da Análise Fatorial com o objetivo de verificar como as medidas de criatividade e inteligência se agrupavam, visto a existência de correlação significativa entre elas. O propósito da análise fatorial nestes casos, seria verificar de que forma se agrupam as medidas de uma bateria de testes que cobre uma diversidade de capacidades intelectuais (neste caso criatividade e inteligência), de forma a identificar subgrupos de testes que avaliam uma mesma capacidade cognitiva (Primi, 2003). Para isso inicialmente o teste de hemisfericidade de Bartllet foi empregado e indicou um valor significativo de $\mathrm{p} \leq 0,0001\left(\chi^{2}=156,48\right.$; $\mathrm{gl}=15$ ) demonstrando a adequação da análise. Assim, a análise fatorial foi empregada, envolvendo a pontuação no desenho da figura feminina e o desenho da figura masculina, além dos quatro fatores da criatividade, sendo que os resultados apontaram, por rotação varimax, a existência de dois fatores que explicariam $61,24 \%$ da variância total $(41,96 \%$ e $19,27 \%$, respectivamente).

A composição final dos fatores indicou a existência de um primeiro fator, composto pela figura feminina (carga fatorial de 0,82$)$, figura masculina $(0,80)$, fator 1 da criatividade - enriquecimento de idéias $(0,80)$ e fator 4 - aspectos cognitivos $(0,56)$. Interessante notar que todas essas medidas constituem-se em medidas cognitivas, tanto da criatividade quanto do desenvolvimento intelectual. A análise indicou um segundo fator que agrupa o fator 2 do teste de criatividade emotividade (carga fatorial de 0,67 ) e o fator 3 - preparação criativa $(0,56)$.

Do ponto de vista teórico, a constatação do agrupamento das medidas em dois fatores faz sentido perante a teoria que embasa o instrumento de criatividade, ao separar as características cognitivas e emocionais que a compõem. Isso porque a literatura tem apontado a necessidade de considerar a avaliação da criatividade dentro de um modelo multidimensional, que incorpore elementos afetivos e emocionais aos cognitivos na avaliação da criatividade, visto que, segundo Sternberg e Grigorenko (2001), os testes cognitivos não predizem a performance criativa muito bem devido ao fato destes testes não levarem em conta as variáveis afetivas e motivacionais. Esta mesma necessidade foi sentida por Torrance, sendo que, após constatar a existência de indicadores emocionais da criatividade expressos nos desenhos que poderiam predizer realizações criativas na vida adulta, ampliou seu modelo de avaliação de maneira que o mesmo deixou de avaliar somente os aspectos cognitivos da criatividade, passando a avaliar também os aspectos emocionais (Wechsler, 2008). Em relação ao instrumento que avalia o desenvolvimento cognitivo, era esperado que suas medidas se agrupassem com os fatores cognitivos do teste de criatividade, o que realmente ocorreu, dado o fato de que, até o momento, o modelo de avaliação desse instrumental tem sido focado nos aspectos cognitivos. Entretanto, novas pesquisas vêm sendo realizadas pela autora com o Teste Desenho da Figura Humana, visando a investigação dos indicadores emocionais, a fim de que os aspectos emocionais contidos nos desenhos sejam também englobados em um novo e mais completo sistema de avaliação.

Em relação ao agrupamento das características cognitivas da criatividade com as medidas cognitivas, vê-se que estudos com objetivos similares são encontrados na literatura, podendo-se citar, como exemplo, a pesquisa desenvolvida por Kershner e Ledger (1985) na qual foram desenvolvidos e também apontaram o agrupamento de algumas características da criatividade com as medidas cognitivas, tal como o relatado por Kershner e Ledger (1985) no qual constatou-se que a inteligência se relaciona a componentes específicos da criatividade (principalmente com a originalidade, flexibilidade e elaboração) e não se encontra relacionada com outros componentes. Análise realizada por Ruth e Birren (1985) também demonstrou que a análise fatorial não produziu dois fatores básicos, um de criatividade e outro de inteligência, conforme esperado, de maneira que, quando os dados foram analisados em relação aos componentes principais, também pode ser notado um agrupamento das medidas. A explicação, de acordo com Russo (2004) amparar-se-ia na constatação de que os testes de criatividade podem ser considerados, de certa forma, um teste de QI, visto que ambos os testes mensuram habilidades cognitivas.

Novas investigações acerca dos motivos que levam o Fator 3 se agrupar no segundo fator devem ser feitas, embora deva-se ressaltar a diferença em sua constituição. A diferenciação deste fator dentro do modelo de correção do instrumento de criatividade se justifica pelo fato de que o mesmo agrupa as características avaliadas na primeira atividade do teste, cuja proposta é bem diferente das outras duas atividades, uma vez que nela solicita-se a realização de um único desenho. Por este motivo é considerado um fator de "aquecimento", uma oportunidade de treino para a realização das atividades posteriores que permitem o uso mais livre da criatividade e um maior número de respostas. Compreender o motivo desse fator ter se agrupado aos componentes emocionais da criatividade merece uma investigação mais aprofundada em estudos posteriores.

Ao se discutir a relação entre as medidas de criatividade e inteligência, duas importantes reflexões devem ser feitas. A primeira se refere à percepção de vários autores sobre o fato de que os testes de criatividade medem um domínio do talento que não é adequadamente representado nos testes convencionais de inteligência (Cropley, 1972), de forma a se reforçar a importância dos dois construtos para o desenvolvimento individual. Dessa maneira um modelo ideal, segundo Sternberg (2001), envolveria a busca pelo equilíbrio entre a necessidade de mudança (possível a partir da presença da criatividade) e a necessidade de estabilidade e continuidade (propiciada pela inteligência). A segunda reflexão refere-se às diferenças encontradas nos estudos. Dependendo de como os construtos são medidos, do instrumento utilizado, da teoria que os embasa, das diferenças metodológicas e da amostra estudada (que varia em função da idade, habilidade 
e nível educacional), os resultados têm apresentado amplas variações (Preckel et al., 2006). Embora a maior parte destes tenha concluído acerca da existência de alguma relação entre criatividade e inteligência, com a amplitude desta relação variando entre baixa e moderada, as diferenças relatadas acabam dificultando o consenso em relação a esta questão.

\section{Considerações Finais}

Espera-se que a presente pesquisa possa colaborar com as discussões ainda inconclusivas acerca da relação entre criatividade e inteligência, visto que, na presente amostra de crianças e adolescentes, esta relação mostrou-se moderada. Pode-se pensar que a complexidade de ambos os fenômenos e as dificuldades para sua avaliação contribuem de maneira significativa para a dificuldade em se obter uma resposta consensual à tentativa de se estabelecer a relação entre os construtos.

Novos estudos com diversificação da amostra e dos instrumentos utilizados são recomendados com a finalidade de fomentar ainda mais as discussões que vêm sendo feitas, de forma que os mesmos poderão confirmar, ou não, os resultados apresentados. Sem dúvida, os resultados de estudos que tenham o objetivo de clarificar as relações entre os construtos poderão contribuir, de forma importante, para os avanços na investigação, por meio da estimulação à realização de novas pesquisas e a busca de novas respostas, ainda que parciais e não consensuais.

Estes estudos poderão ser de grande valor, principalmente quando se discute a questão da superdotação devido à temática em foco da superdotação. Considerando que este fenômeno se compõe de aspectos relacionados à inteligência, criatividade e aspectos motivacionais, estabelecer a relação entre cada um desses componentes se torna importante na medida em que esse conhecimento poderá auxiliar uma melhor identificação desses indivíduos a fim de que seus talentos sejam melhor aproveitados e desenvolvidos.

\section{Referências}

Aguirre, K., \& Conners, F. (2010). Creativity and intelligence in preschoolers: preliminary findings. The University of Alabama McNair Journal. Retrieved from http:/graduate.ua.edu/ mcnair/journals/2010/Aguirre.pdf.

Aranha, M. A. R. C. (1997). Creativity in students and its relation to intelligence and peer perception. Revista Interamericana de Psicologia, 31(2), 309-313.

Bandeira, D. R., Costa, A., \& Arteche, A. (2008). Estudo de validade do DFH como medida de desenvolvimento cognitivo infantil. Psicologia Reflexão e Crítica, 21, 332-337.

Barros, D. P., Primi, R., Miguel, F. K., Almeida, L., \& Oliveira, E. P. (2010). Metaphor creation: A measure of creativity or intelligence? European Journal of Education and Psychology, 3(1), 103-115.

Batey, M., Furnham, A., \& Safiullina, X. (2010). Intelligence, general knowledge and personality. Learning and Individual Differences, 20, 532-535.
Boden, M. A. (1999). Dimensões da criatividade. Porto Alegre: Artes Médicas.

Brunner, M., Krauss, S., \& Kunter, M. (2008). Gender differences in mathematics: Does the story need to be rewritten?.Intelligence, 36, 403-421.

Camarata, S., \& Woodcock, R. (2006). Sex differences in processing speed: Developmental effects in males and females. Intelligence, 34(3), 231-252.

Candeias, A. A. (2008). Criatividade: Perspectiva integrativa sobre o conceito e a sua avaliação. In M. F. Morais \& S. Bahia (Eds.), Criatividade: Conceito, necessidades e intervenção (pp. 4164). Braga, Portugal: Psiquilíbrios.

Chiodi, M. G., Nakano, T. C., \& Wechsler, S. M. (2011a). Estudo de correlação entre criatividade e habilidades intelectuais. Trabalho apresentado no I Congresso Internacional de Criatividade. Inovação, Manaus, Amazonas.

Chiodi, M. G., Nakano, T. C., \& Wechsler, S. M. (2011b). Inteligência e criatividade: um estudo correlacional. Anais do I Congresso Internacional de Criatividade. Inovação. Trabalho apresentado no I Congresso Internacional de Criatividade. Inovação, Manaus, Amazonas.

Cropley, A. J. (1972). A five-year longitudinal study of the validity of creativity tests. Developmental Psychology, 6(1), 119-124.

Dai, X., Ryan, J.J., Paolo, A.M. \& Harrington, R.G. (1991). Sex differences on the Wechsler Adult Intelligence Scale- Revised for China. Psychological Assessment, 3 (2), 282-284.

De La Torre, S. (2005). Dialogando com a criatividade. São Paulo: Madras.

Edwards, M. P., \& Tyler, L. E. (1965). Intelligence, creativity and achievement in nonselective public junior high school. Journal of Educational Psychology, 56(2), 96-99.

Elisondo, R. C., \& Donolo, D. S. (2010). ¿Creatividad o inteligencia? That is not the question. Anales de Psicología, 26(2), 220-225.

Eysenck, H. (1999). As formas de medir a criatividade. In M. A. Boden (Ed.), Dimensões da criatividade (pp. 203-225). Porto Alegre: Artes Médicas.

Feldman, D. H. (2000). The development of creativity. In R. J. Sternberg (Ed.), Handbook of creativity (pp. 169-189). Cambridge: Cambridge University Press.

Gagné, F. (2005). From gifted to talents: The DMGT as a developmental model. In R. J. Sternberg, \& J. E. Davidson (Eds.), Conceptions of Giftdeness (pp. 98-120). New York: Cambridge University Press.

Getzels, J. W., \& Jackson, P. W. (1962). Creativity and intelligence: Explorations with gifted students. New York: John Wiley \& Sons.

Goldbeck, L., Daseking, M., Hellwig-Brida, S., Waldmann, H.C. \& Petermann, F. (2010). Sex differences on the German Wechsler Intelligence Test for Children (WISC-IV). Journal of Individual Differences, 31(1), 22-28.

Hattie, J., \& Rogers, H. J. (1986). Factor models for assessing the relation between creativity and intelligence. Journal of Educational Psychology, 78(6), 482-485.

Irwing, P. (2012). Sex differences in g: an analysis of the US standardization sample of the Wais-III. Personality and Individual Differences, 53, 126-131.

Jarosewich, T., Pfeiffer, S. I., \& Morris, J. (2002). Identifying gifted students using teacher rating scales: a review of existing instruments. Journal of Psychoeducational Assessment, 20, 322-336. 
Kaufman, S. B. \& Sternberg, R. J. (2008). Conceptions of giftedness. In S. Pfeiffer (Ed.), Handbook of giftedness in children: Psycho-educational theory, research and best practices. (pp. 71-91). New York: Springer.

Kershner, J. R., \& Ledger, G. (1985). Effect of sex, intelligence, and style of thinking on creativity: A comparison of gifted and average IQ children. Journal of Personality and Social Psychology, 48(4), 1033-1040.

Kim, K. H., Cramond, B., \& Bandalos, D. L. (2006). The latent structure and measurement invariance of scores on the Torrance tests of Creative Thinking-Figural. Educational and Psychological Measurement, 66(3), 459-477.

Kneller, G. F. (1971). Arte e ciência da criatividade. São Paulo: Ibrasa.

Lubart, T. (2007). Psicologia da criatividade. Porto Alegre: ArtMed.

Lundsteen, S. W. (1966). Book reviews: Modes of thinking in young children: a study of creativity-intelligence distinction by Michael A. Wallach and Nathan Kogan. Educational and Psychological Measurement, 26, 523-527.

Maia-Pinto, R. R., \& Fleith, D. S. (2004). Avaliação das práticas educacionais de um programa de atendimento a alunos superdotados e talentosos. Psicologia Escolar e Educacional, $8(1), 55-66$.

Montuori, A., \& Purser, R. E. (1995). Deconstructing the lone genius myth: Toward a contextual view of creativity. Journal of Humanistic Pyschology, 35(3), 69-112.

Mouchiroud, C., \& Lubart, T. (2002). Social creativity: A crosssectional study of 6 to 11 year-old children. International Journal of Behavioral Development, 26(1), 60-69.

Nakano, T. C. (2003). Criatividade Figural: proposta de um instrumento de avaliação. Dissertação de Mestrado, Pontifícia Universidade Católica de Campinas. Campinas, SP.

Nakano, T. C., \& Brito, M. E. (no prelo). Avaliação da criatividade a partir do controle do nível de inteligência em uma amostra de crianças.

Nakano, T. C., \& Wechsler, S. M. (2006a). Teste Brasileiro de Criatividade Figural: proposta de instrumento. Revista Interamericana de Psicologia, 40, 103-110.

Nakano, T. C., \& Wechsler, S. M. (2006b). Teste Brasileiro de Criatividade Figural: proposta de normas. Avaliação Psicológica, 5(2), 159-170.

Nakano, T.C., Wechsler, S. M., \& Primi, R. (2011). Teste de Criatividade Figural Infantil. São Paulo: Vetor.

Nisbett, R.E,, Aronson, J., Blair, C., Dickens, W., Flynn, J., Halpern, D.F. \& Turkheimer, E. (2012). Intelligence: findings and theoretical developments. American Psychologist, 67 (2), 130-159.

Nogueira, S. M., \& Pereira, M. (2008). Estratégias promotoras da criatividade. In M. F. Morais e S. Bahia (Eds.), Criatividade: conceito, necessidades e intervenção (pp. 253-278). Braga, Portugal: Psiquilibrios.

Nusbaum, E. C., \& Silvia, P. J. (2011). Are intelligence and creativity really so different? Fluid intelligence, executive processes, and strategy use in divergent thinking. Intelligence, 39, 36-45.

Preckel, F., Holling, H., \& Wiese, M. (2006). Relationship of intelligence and creativity in gifted and non-gifted students: An investigation of threshold theory. Personality and Individual Differences, 40, 159-170.
Primi, R. (2003). Inteligência: avanços nos modelos teóricos e nos instrumentos de medida. Avaliação Psicológica, 2(1), 67-77.

Rammstedt, B. \& Rammsayer, T.H. (2002). Self-estimated intelligence: gender differences, relationship to psychometric intelligence and moderating effects of level of education. European Psychologist, 7 (4), 275-284.

Renzulli, J. S. (2008). La educación del sobredotado y el desarrollo del talento para todos. Revista de Psicologia (Lima), 26(1), 25-44.

Rindermann, H., \& Neubauer, A. C. (2004). Processing speed, intelligence, creativity, and school performance: Testing of causal hypotheses using structural equation models. Intelligence, 32, 573-589.

Runko, M. A., \& Mraz, W. (1992). Scoring divergent thinking tests using total ideational output and a creativity index. Educational and Psychological Measurement, 52, 213-221.

Russo, C. F. (2004). Comparative study of creativity and cognitivesolving strategies of high-IQ and average students. Gifted Child Quarterly, 48(3), 179-190.

Ruth, J., \& Birren, J. (1985), Creativity in adulthood and old age: Relations to intelligence, sex and mode of testing. International Journal of Behavioral Development, 8, 99-109.

Santos, A. A. A., Noronha, A. P. P., \& Sisto, F. F. (2005). Teste de inteligência R1-forma B e G36: Evidência de validade convergente. Estudos de Psicologia (Natal), 10(2), 191-197.

Savage-McGlynn, E. (2012). Sex differences in intelligence in younger and older participants of the Raven's standard progressive matrices plus. Personality and Individual Differences, 53, 137-141.

Shaffer, D. R. (2005). Psicologia do desenvolvimento. São Paulo: Editora Thomson Learning.

Silvia, P. J. (2008a). Another look at creativity and intelligence: Exploring higher-order models and probable confounds. Personality and Individual Differences, 44, 1012-1021.

Silvia, P. J. (2008b). Creativity and intelligence revisited: A latent variable analysis of Wallach and Kogan (1965). Creativity Research Journal, 20(1), 34-39.

Sternberg, R. J. (1984). What should intelligence tests test? Implications of a triarchic theory of intelligence for intelligence testing. Educational Researcher, 13, 5-15.

Sternberg, R. J. (2001). What is the common thread of creativity? Its dialectical relation to intelligence and wisdom. American Psychological Association, 56(4), 360-362.

Sternberg, R. J. (2006). Creating a vision of creativity: The first 25 years. Psychology of Aesthetics, Creativity, and the Arts, $\mathrm{S}(1), 2-12$.

Sternberg, R. J., \& Grigorenko, E. L. (2001). Guilford's structure of intellect model and model of creativity: contributions and limitations. Creativity Research Journal, 13(3/4), 309-316.

Sternberg, R. J., \& O'Hara, L. A. (2000). Intelligence and creativity. In R. J. Sternberg (Ed.), Handbook of intelligence (pp. 611630). New York: Cambridge University Press.

Torrance, E. P. (1976). Criatividade: medidas, testes e avaliações. São Paulo: Ibrasa.

Torrance, E. P. (1980). Creative intelligence and "an agenda for the 80's." Art Education, 33, 8-14.

Wallach, M. A., \& Kogan, N. (1965). Modes of thinking in young children. New York: Holt, Rinehart \& Winston. 
Wechsler, S. M. (2003). DFH III: O desenho da figura humana: avaliação do desenvolvimento cognitivo infantil ( $3^{\text {a }}$ edição ampliada e atualizada). Campinas: Impressão Digital do Brasil.

Wechsler, S. M. (2008). Criatividade: descobrindo e encorajando. São Paulo: Editora Psy.

Wechsler, S. M., Nunes, M. F. O., Schelini, P. W., Ferreira, A. A., \& Pereira, D. A. P. (2010). Criatividade e inteligência: analisando semelhanças e discrepâncias no desenvolvimento. Estudos de Psicologia (Natal), 5(3), 243-250.

Wechsler, S. M., \& Richmond, B. (1984). Influências da dotação intelectual e criativa no ajustamento em sala de aula. Arquivos Brasileiros de Psicologia, 36(2), 138-147.
Wechsler, S. M., \& Schelini, P. W. (2006). Bateria de habilidades cognitivas Woodcock-Johnson III: validade de construto. Psicologia: Teoria e Pesquisa, 22(3), 287-296.
Recebido em 25.05.2010

Primeira decisão editorial em 09.07.2011

Versão final em 21.07.2011

Aceito em 15.09.2011 